\title{
Polysemy derivation in a multidimensional framework
}

\author{
Antonio José Silvestre-López \\ Universitat Jaume I de Castelló \\ asilvest@uji.es
}

\begin{abstract}
The aim of this paper is to systematise and illustrate the process of semantic network derivation within the multidimensional polysemy framework (Navarro, 1998, 2006) by way of a detailed analysis of the preposition into. The theoretical and methodological proposals introduce a series of parameters devised to identify, discriminate and classify network senses that can be applied to any preposition analysed within this framework. The results of the process (based on the manual analysis and classification of corpus examples) are illustrated by a description of the primary and secondary senses with which into is commonly associated in discourse as arranged in a multidimensional network.
\end{abstract}

Keywords: multidimensional polysemy, sense derivation, procedures, prepositions, into

\section{Introduction}

Prepositional semantics has been extensively studied from a number of perspectives; along the past decades, cognitive linguistics has yielded diverse proposals to depicture the organisation of spatial meaning in terms of, for example, radial (Brugman, 1980; Lakoff, 1987), hierarchical (Langacker, 1987) and multidimensional (Deane, 2005; Geeraerts, 2007) networks. ${ }^{1}$

Spatial relationships have been portrayed in a variety of fashions along recent approaches to prepositional semantics in terms of three configurational aspects that derive directly from our sensory-motor and functional experience: topology, force- 
dynamics, and function. ${ }^{2}$ Albeit with a different labelling system, the importance of these three dimensions in the semantic configuration of particles has been attested with psycholinguistic evidence in a series of studies (Cf. for example, Coventry and Garrod, 2004 or Feist, 2000, 2004). From a linguistic perspective, some of the most widely known approaches include, for example, Vandeloise's $(1991,2003)$ work on Complex Primitives or Tyler and Evans's (2003) Principled Polysemy Framework, which can be integrated into the more comprehensive Lexical Concepts and Cognitive Models Theory (Evans, 2009). The study presented in this paper embraces a slightly evolved model for Multidimensional Polysemy (Navarro, 1998, 2006), which integrates the three construal aspects within multidimensional radial networks (Cf. Silvestre-López, 2009 for a review).

The model proposes that the relationships between Trajector (TR) and Landmark (LM) in a given situation - as perceived by humans - are processed and integrated into a particular configuration of topological, dynamic ${ }^{3}$ and functional aspects. This configuration sets the ground for the construal of each situation (it often influences the speakers' linguistic choices within the lexical repertoire) and is, in this model, represented in terms of multidimensional radial networks. The specific conflation of these parameters has a bearing not only on the arrangement of senses along the network but also on the determination of their nature. Navarro (2006: 171) describes each parameter as follows:

1- Topology: The visual perception of objects gives the speaker clues for establishing and conceptualising topological relations like coincidence, contact, inclusion, proximity, and the like.

2- Dynamics: Human beings have experience of self-motion and object motion, which provides the clues for conceptualising patterns of interaction in terms of dynamics.

3- Function: Human beings have experience of the effects of interaction, as well as the consequences of those effects for survival and well-being. This kind of experience may be projected onto other entities -animals and objects- so that the functional patterns conceptualised on the basis of human interaction are also used for the conceptualization of spatial relationships between other entities.

1.1. Construal aspects, senses and semantic networks in the Multidimensional Polysemy approach

Departing from the level of perception, human cognitive processing of perceptual space involves the simultaneous perception and processing of a scene in terms of the three construal dimensions (Navarro 2006); although these three aspects are commonly cut apart for practical analytical purposes, they occur simultaneously in our cognitive reality. Every sense in this framework is defined in terms of a particular configuration of these construal features. This particular construal configuration, in turn, is associated with a schematic representation, a structural layout portraying the most outstanding relationships between TR and LM in terms of these features. ${ }^{4}$ 
The primary member within the semantic network proposed for a preposition, that is, that from which other senses can be derived and explained is labelled protoconcept (Navarro, 2006: 173). Both protoconcepts and derived senses are multidimensional in that the three dimensions or aspects of construal are always present and play a role in 'construing' every particular configuration. The particular contribution of each construal aspect, however, may vary depending on the of TR-LM relationship inherent in a given scene.

The variety of ways in which the three dimensions may combine to yield a particular construal configuration associated with a sense is coded in terms of the distribution of senses along the regions or dimensions of a network. This can be better perceived with the help of Figure 1, which shows a template of regions for sense distribution upon which a radial network can be built. ${ }^{5}$ Each region graphically represents either a single construal dimension (topology, dynamics, function) being emphasised (its contribution to the whole construal configuration is more salient) or a combination of emphasised construal dimensions.

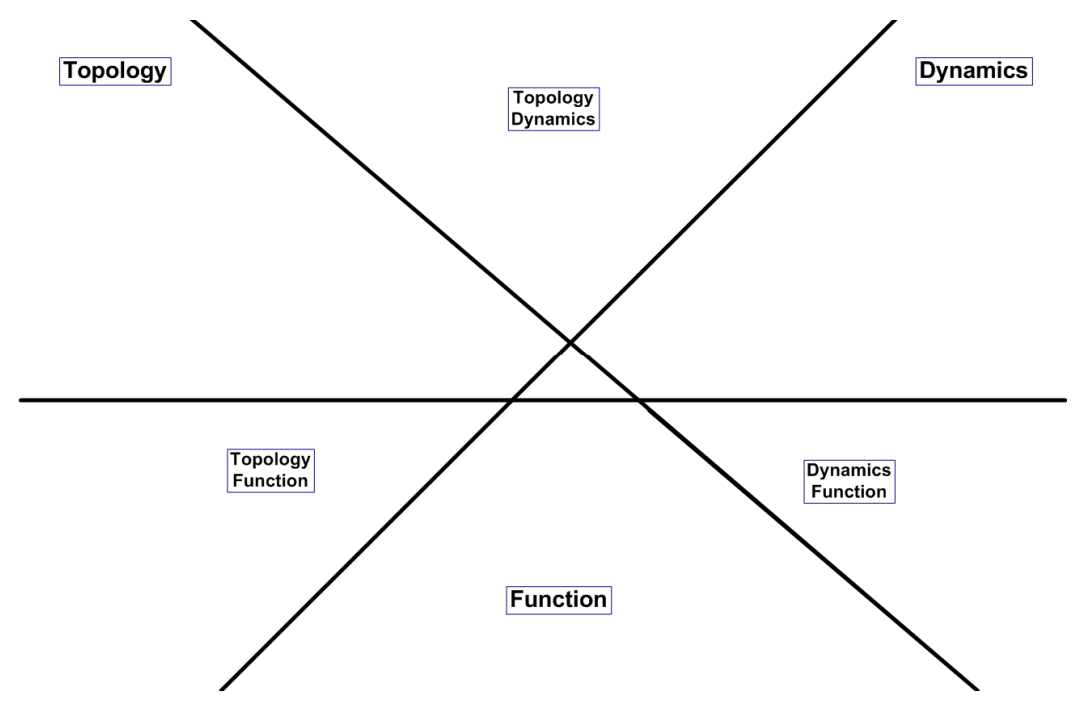

Figure 1. Sense distribution layout for network creation

The three construal dimensions are configured in a balanced relationship (equal importance in terms of contribution) at the very centre of the network. The remaining six regions represent cases where one or two construal dimensions are more salient. Thus, any sense located in any of these areas is assumed to exhibit an 'unbalanced' configuration in such a way that either one or two aspects become more salient than the rest in the construal of the situation.

The role of each of these dimensions is combined with a series of processes involved in the derivation of senses. Navarro $(1998,2006)$ suggests some of these processes, for example, shifts in the original conceptualisation pattern of the primary sense, profiling of particular elements, ${ }^{6}$ metaphoric projections, or semantic bleaching. Although these processes have been identified (Navarro, 1998, 2006), their application 
(how they may combine and interact) has not been systematised yet in the process of sense analysis and derivation. The present study intends to refine the multidimensional polysemy model in this respect.

\subsection{The contribution of this study}

Tyler and Evans' (2003) principled polysemy framework proposes a methodology for sense identification and classification which, albeit intended to be replicated in further polysemy derivation studies, applies under their framework. While acknowledging Tyler and Evans' valuable contribution to the field, the present study - being 'framed' in a different model - proposes likewise a further series of sense discrimination criteria developed specifically from the parameters that drive polysemy derivation under a different framework of multidimensional polysemy.

More concretely, the main purpose of this paper is to describe the process and product of the derivation of multidimensional semantic networks under Navarro's Framework of Multidimensional Polysemy in a systematised way. This description is illustrated through the derivation of the senses with which into is used in discourse.

In this view, the process described (methodology) is as important as the product (results) itself. The method section, on the one hand, sets forth a series of newly systematised procedures ${ }^{7}$ (example classification and analysis in terms of a set of contextual parameters and a series of sense identification and discrimination criteria) to be followed so as to 'build' multidimensional semantic networks for any particle under this framework. The results and discussion section, on the other hand, illustrates this process with the case of into, presents the resulting multidimensional network, and provides an extended description of the primary sense followed by a sketch of the main features of each derived sense that highlights the variations that allowed their presence in the network

The purpose and considerations mentioned above can be narrowed down into two well-defined methodological aims - that is to say, two aims that drive the methodology developed and the results obtained:

AIM 1: Detection of the range of (sets of) uses of into in discourse.

Once detected, these sets of uses (discursive behavioural patterns of into) yield an initial (unpolished) array of sense profiles (see below).

AIM 2: Determination of the nature of each sense profile and (proper) sense arrangement within the multidimensional radial network.

\section{Methodology}

The procedures followed to derive the semantic network for into are presented here as organised in terms of each of the aims above. ${ }^{8}$ 


\subsection{AIM 1: Methodological considerations and procedures}

The methodology adopted to detect the range of uses of into in general discourse and to identify recurrent usage patterns involves an exhaustive analysis of real examples and their classification into sense profiles.

A sense profile, as conceived of in this study, may be defined as a set of uses of a preposition with a series of common features. A sense profile in this respect is based on an embryonic set of configurational features that define a potential proper sense. ${ }^{9}$ The nature of these features is unveiled as the manual analysis of examples proceeds; thus, the more examples analysed, the wider the perspective on the behaviour of into in discourse. The construction of each profile involves not only a classification of examples but also an initial determination of the most salient patterns of construal configurations, an initial draft of a structural layout and the identification of a series of associated contextual aspects.

In order to detect the different sense profiles of into, a semantic analysis of 1,000 randomly-extracted British National Corpus (BNC) examples was carried out. One previous/subsequent sentence was allowed for each example so as to provide for the necessary contextual information. The examples were then analysed manually and gathered in terms of similarities so as to obtain an initial list of sense profiles.

Context plays a crucial role in the identification and classification of sense profiles. In order to allow for the contextual dimension, the following series of parameters was observed and recorded in the manual analysis of each example:

1. TR-LM animacy conditions, coded in a scale from human beings to fixed objects. Animacy conditions (Feist, 2000, 2004) of the participants involved in the relationship may be a hint for possible conceptualisations of dynamic effects and functional consequences, hence their inclusion as the first parameter.

2. Type of domain for conceptualisation. The identification of typical domains shows broad areas or general contexts of use, hence allowing a wider perspective where representative TRs and LMs may be predicted.

3. Literal vs. metaphoric uses, where this distinction proves important in licensing specific uses and in determining particular kinds of TRs and LMs associated with them.

4. Other outstanding sentential context features: e.g. prolific verbal elements.

5. Construal configuration: information on the peculiarities of the TR-LM relationship as portrayed in the example, coded in terms of the effects of the combined contribution of the three construal aspects found in the example. This is usually accompanied by a 'sense profile label' (e.g. 'ENTRY') to quickly identify the example with a particular kind of construal configuration that might also be found in other examples.

Although each profile might eventually be considered as a proper sense, at this stage it only seems safe to talk about particular 'sets of uses' that can be gathered under 
a profile. In other words, some of the uses identified as such at this point might actually and eventually (after the application of the criteria suggested below) simply rank as contextual uses or variations of a particular sense per se, as opposed to cases of proper senses (primary or derived) with network node status (i.e. properly established senses reflected as such on the network).

\subsection{AIM 2: Methodological considerations and procedures}

Once a set of sense profiles has been identified, the next logical step is to determine the nature of each of them. This involves identifying the sense profiles as primary or secondary (i.e. proper) senses or else as contextual variations of a proper sense.

A series of parameters for the identification of senses and establishing objective distinctions among them were set as a methodological aid for the researcher; these involve, on the one hand, a set of features with which the primary sense is commonly associated (section 2.2.1) and on the other hand, a set of criteria involved in the identification and distinction among derived senses (section 2.2.2). The latter were also devised to avoid network overpopulation by preventing sense profiles that conceal mere contextual variations - that is, the examples they encompass show plain contextual effects that actually derive from the structure of a different, properly established sense from being considered as proper senses.

The discrimination of contextual uses is inherent to the identification of primary and secondary senses and entails a process of rearrangement (subsumption) of the sets of examples associated with a 'contextual variation sense profile' as cases of a different in this case fully-established - proper sense. This process is further developed and illustrated with the case of into in section 3.1.

At this point the reader may have noted that determining the nature of the senses of into is actually an 'online' process that develops as the methodological procedures proposed for example analysis and classification are followed. The picture of the uses of into in discourse therefore becomes more and more complete as the process of analysis proceeds.

\subsubsection{Features of the primary sense}

The protoconcept is assumed to show an essential configuration along the range of senses associated with a preposition that is semantically rich enough to allow for sense derivation. The primary sense is therefore conceived of as the primigenial one in that it provides the basis for meaning specialisation (i.e. the derivation of secondary senses) and extension (e.g. projections yielding metaphorical uses of an original configuration).

The degree of entrenchment of a linguistic unit has been associated with the frequency with which an individual or a speech community use it as associated with a particular meaning (Langacker, 1987). The frequency with which a unit is used with a particular meaning in a corpus, as compared with the absolute set of alternative meanings with which the unit is used in the corpus, has been proved to correlate with 
the degree of entrenchment (onomasiological salience) of that unit-meaning (Geeraerts et al., 1994; Navarro, 1998).

In the light of these considerations, an association between the primary sense and the most salient of the whole set of senses may be established. The experience acquired in the derivation of multidimensional networks for different prepositions under this framework (e.g. Navarro, 1998, 2006; Navarro and Gösser, 2011; the present work) points to a tendency for the sense eventually identified as the protoconcept to actually show the most basic schematic layout of the TR-LM relationship, which comes associated with a particular construal aspect configuration. It is precisely its basic configuration - which might in turn explain its salience over the rest of senses - which renders a ready-to-use 'material' from which other senses may develop. A combination of two criteria is thus suggested to help identify the primary sense:

$\alpha$ - The sense profile showing the highest frequency rate among the range of sense profiles with which a unit (preposition) is associated.

$\beta$ - The sense profile displaying the most basic schematic configuration.

$\alpha$ and $\beta$ are understood here as complementary features upon which a 'compromise' should be reached. In most cases both of them coincide in the same sense, which can be taken as an indicator of a good 'protoconcept candidate'. If a case is given where $\alpha$ is met by one sense and $\beta$ is met by another one, this implies that there are two prototypical uses of a preposition, each with one basic feature. The two of them are basic and active at a point in time (current speech), but only one of them (that identified with $\beta$ ) qualifies as the protoconcept.

Once the primary sense has been identified, both its particular structural layout and construal configuration can be taken as the reference set of features against which the rest of senses (profiles showing significantly different configurations) may be compared. Important variation in these terms can therefore lead to the consideration of a particular sense profile as being derived from the primary one. This kind of variation is reflected in the sense discrimination criteria.

\subsubsection{Distinction of derived senses}

The senses in the network are kept to a minimum and are distinguished by way of the application of the following discrimination criteria:

\section{A. Transformations in the original structure of the primary sense}

While sharing some of their structure, criterion A suggests that any transformation in the original schematic structure (basic layout) of the primary sense may yield a different sense. Peña and Ruiz de Mendoza (2009) defend the metonymic grounding of imageschema transformations like path-end-of-path (Lakoff, 1987) by suggesting that instead of a complex transformation the phenomenon can be explained more parsimoniously 
through a metonymic focus on one of the path structural elements. This study applies their proposals to protoconcepts. Thus, the transformations described here are often explained via underlying metonymic projections upon parts of the schematic structure of the primary sense, resulting in highlighted or downgraded parts or elements.

Additionally, the basic layout of the primary sense may be 'enriched' with additional TR-LM features. In these cases, the transformation is the product of an underlying metaphorical projection (criterion C).

\section{B. Modifications in the original configuration of construal aspects}

This criterion makes reference to any kind of variation in the original structure whose effects show a construal aspect configuration that is different from the primary sense (or a mother network node arising from the primary sense). In this view, a different pattern of emphasised (salient) construal aspects may be indicative of a different sense.

\section{Metaphoric licensing}

One or a combined set of metaphoric mappings - often primary metaphors (Grady, 2007) and/or realisations of the event structure metaphor (Lakoff, 1987) within welldefined contexts of use - motivate the existence of a sense in the network. These mappings bring about with them a transformation in $\mathrm{A}$ and/or a different configuration in B above, i.e. the presence of metaphor licenses the application of A or B. All corpus examples fitting the sense configuration are therefore necessarily metaphorical in nature.

Secondary proper senses must meet both criteria A and B. Because of the changes it brings about, criterion $\mathrm{C}$ necessarily happens with $\mathrm{A}$ and $\mathrm{B}$. C is not as primary as $\mathrm{A}$ or $\mathrm{B}$ in terms of frequency and application, but it is crucial in determining the status of sense profiles where metaphor is involved (e.g. as metaphorically-licensed proper senses vs. sets of metaphorical extensions of proper senses).

\section{Results and discussion}

The 'final product' of the procedures described above is the multidimensional radial network of into. The network itself is introduced in section 3.3 followed by a detailed description of the primary sense (3.4.) and an account of the main features of secondary senses (3.5). Before diving into the network as such, however, section 3.1. briefly illustrates the sense profile classification process of into (resulting from the procedures described under 2.1), and section 3.2 the identification of its primary sense (resulting from 2.2.1). The application of secondary sense discrimination criteria (resulting from 2.2.2.) is provided along with the secondary sense description. ${ }^{10}$ 


\subsection{On sense profiles}

9 different sense profiles were identified along the first stages of corpus example analysis, namely: ENTRY, ENCLOSURE COLLISION, TR BECOMES A GROUP, LM CONSTRAINS TR'S CONTROL, ${ }^{11}$ DIRECTION (e.g. looking/peeping into the LM), TR BECOMES AN INTEGRATED PART OF LM (e.g. 'building' or 'melting' the TR into a larger complex unit (LM) so that it becomes an integrated part of it), FORCEFUL ENTRANCE (e.g. the TR 'breaks into' the LM) and LM CONSTRAINS TR'S MOTION INSIDE (e.g. the TR is forced into the LM in a way that it cannot move within or out of it).

Among them, ENTRY was identified as the primary sense (see below), the next four were found to meet at least criteria $\mathrm{A}$ and $\mathrm{B}$, and the rest did not show significant differences (one or no criteria met) and were therefore dismissed as proper senses; the latter set, however, were observed to conceal contextual uses of proper senses and were rearranged accordingly as such into a network-status sense. Providing a detailed account on the intricacies of each rearrangement case falls beyond the scope of this paper, but a brief illustration of two examples may help the reader to get a clearer idea on the process.

The DIRECTION sense profile was found to gather a series of uses where the TR, an animate entity, is located outside the LM and is looking, peeping, or gazing into it. Initially this set had been conceived of as a potential proper sense owing to the presence of a fine-grained series of recurrent elements (e.g. contextual parameters like 'look/peer'-like verbs, no motion implied, etc.); however, none of the discrimination criteria were found to apply, which allowed considering this sense profile as a particular set of uses within the ENTRY sense, more concretely as one of the metaphorical extension $^{12}$ sets of ENTRY motivated by the seeing is entering metaphor (Navarro, 1998: 254) in combination with a metonymic projection of the sense of sight for the whole experiencer.

Along this process, it is rather frequent for a sense profile to meet only one criterion; in this case the differences with respect to a more primary node are not significant enough for it to be granted network status. This is the case of the FORCEFUL ENTRANCE profile, which encompasses senses where the TR 'breaks into' the LM, a fully-closed container. While there is a variation in the schematic layout, the construal configuration pattern of ENTRY is fully kept. These differences, not being significant enough, led the cases included in FORCEFUL ENTRANCE to be subsumed in the ENTRY sense). 
3.2. Primary sense identified

\begin{tabular}{|l|c|}
\hline \multicolumn{1}{|c|}{ Sense } & Frequency (\%) \\
\hline ENTRY & 75.6 \\
\hline LM CONSTRAINS TR'S CONTROL & 11 \\
\hline COLLISION & 6,1 \\
\hline TR BECOMES A GROUP & 4,6 \\
\hline ENCLOSURE & 2,7 \\
\hline
\end{tabular}

Table 1. Proper sense frequency out of corpus examples

Table 1 shows the percentages resulting from the classification of senses after the rearrangement process. Different effects derive from these data. While the highest value shows a clear salience of the configuration associated with ENTRY in the uses of into, lower percentages might be hinting at potential overlaps of the uses of into with which they are associated with those of a different preposition within the English language system. This seems especially likely for ENCLOSURE, whose configuration may be a borderline use of into, but central among the senses of prepositions like in - in other words, this configuration is more prototypically coded in English by in than into (Cf. Navarro, 1998: 227-230).

As for the determination of the primary sense, in the case of into, ENTRY was found to meet both primary sense criteria: ranking as the most frequent $(\alpha)$ and also showing the most basic configuration $(\beta)$ among the rest of senses (Cf. description in section 3.4).

\subsection{Semantic network}

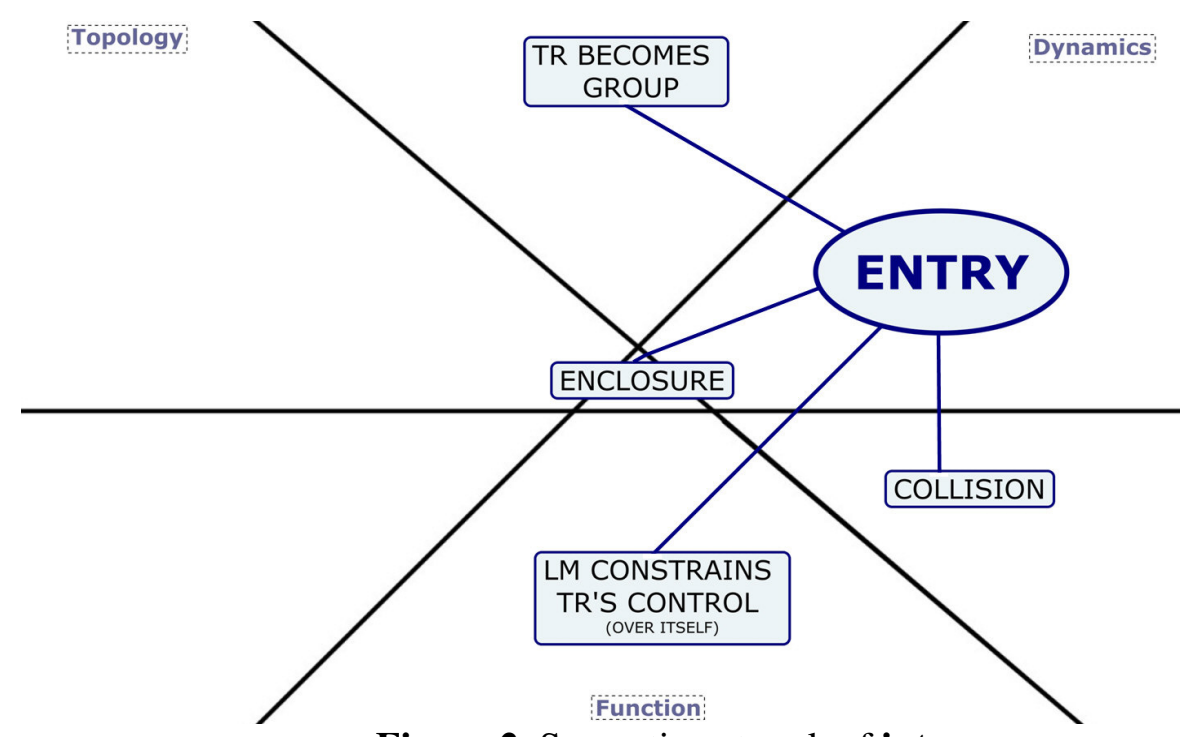

Figure 2. Semantic network of into

Figure 2 represents the multidimensional radial network of into resulting from our analysis. The primary sense for into is not placed at the very core of the diagram (a 
secondary sense occupies instead this position) but within the dynamics region. This is due to the significant role of the dynamic component (in terms of motion and/or force) of the primary sense (Dewell, 2005).

From the overall distribution of senses, dynamics can also be perceived as a pervasive and salient component (to different degrees) along the network; the fact that the 'heavier' or more populated parts of the network are closer to the dynamics region is no mere coincidence.

Finally, before delving into the description of the primary and secondary senses, it is important to note here that when one specific kind or pattern of construal aspects is emphasised (e.g. dynamics, or dynamics plus function), the rest (e.g. topology and function, or topology) still contribute to the conceptualisation of the scene, only that they are not salient in that particular configuration, as associated with a specific use of into. Thus, while the dynamics dimension is also present in, say, the LM CONSTRAINS TR'S CONTROL OVER ITSELF sense of into, its contribution to the whole construal configuration is 'less significant' than the functional implications of the relationship.

\subsection{Primary sense described: ENTRY}

The protoconcept for into is here labelled ENTRY after Dewell (2005), as the label encompasses the basic components of the relationship in an utterly dynamic configuration. In fact, I share most of my views with this author in terms of stative and active configurations of the elements involved in the notion of containment (Cf. Dewell, 2005: .374-378 for an in-depth description). The label is also appropriate in that it encapsulates a boundary-crossing component (Tutton, 2009) inherent in the primary sense.

The description of ENTRY presented here is based on: an account of (i) the main features of the TR-LM configuration, (ii) the multidimensional construal aspects of ENTRY and (iii) contextual information parameters. ${ }^{13}$

It is important to note that the descriptions of ENTRY - as much as those of the rest of senses - provided here are intended to reflect generic features associated with each sense as abstracted out from particular instance analysis. These descriptions are illustrated with a series of examples which, in this light, must be conceived of as representative instantiations (particular realisations) of the more general and encompassing features purported in each sense. The following are some of these representative examples of ENTRY:

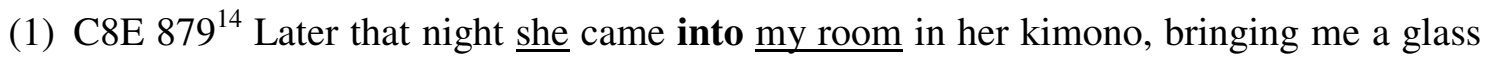
of champagne and carrying a book.

(2) KS7 479 I've got to go into hospital on erm well, tomorrow actually for erm another operation erm...

(3) J1C 1049 I couldn't see it too clearly 'cos it was at the other end of the pitch, but it seemed that Jamie had the ball around the right hand edge of the box and curled it into the top left hand corner. 


\subsubsection{The basic TR-LM configuration}

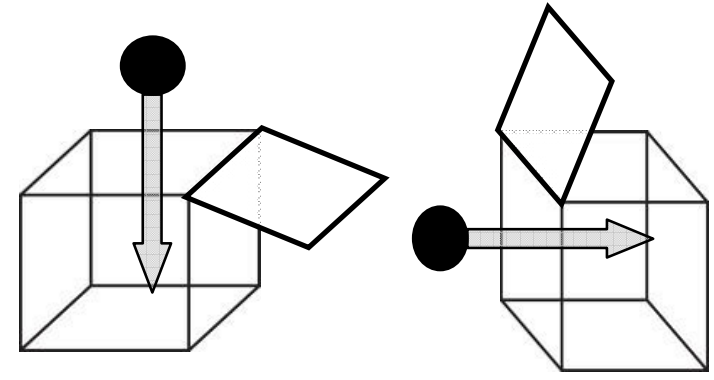

Figure 3. Basic TR-LM configuration of ENTRY

Figure 3 is a schematic representation of the basic configuration of TR and LM of ENTRY. This information can be completed with a series of features that tend to be present in TRs and LMs. ${ }^{15}$

The TR is typically a mobile, finite entity able to be contained within a different entity, the LM. It is canonically smaller than the LM and is to follow path whose endpoint is the interior of the LM.

The LM is typically an entity that defines the boundaries of a three-dimensional region and so determines the limits and capacity of that region. Canonically, it is a container whose boundaries are incomplete. The LM is open on one of its sides (upper or lateral, hence the double schema represented in Figure 3) so that an access exists through which entities (TRs) may enter or exit. The LM's 'access side' might possibly be closed at a previous or later point in time (e.g. at initial or final stages of the relationship, see below).

\subsubsection{Multidimensional construal aspects of ENTRY}

Owing to the dynamic configuration of ENTRY, the description of its multidimensional construal aspects implies a conceived situation where a change of position or a change of state of the entities involved (LM as much as TR) also entails a period of time where an initial, a middle and a final stage or position can be distinguished and observed. This also applies to other derived senses in a way that different peripheral senses may highlight or describe only some of these three specific stages. The reader might like to conceive of the first kind of cases as instantiations of Langacker's (1987) notion of summary scan, where the conceptualisation of a middle or final stage also implies a previous series of changes in the TR-LM sequence, and the second kind (e.g. explicit metonymic focus on one stage) as sequential scan instantiations.

The following is a basic description of the construal aspect configuration of ENTRY. It is actually an indivisible whole, but it has been broken down here into each aspect for the sake of clarity. Each construal aspect, in turn, includes a description of the main traits of the TR-LM relationship in each relevant position or stage. ${ }^{16}$ 


\section{TOPOLOGY}

- Initial position or stage:

Initially, the TR is located outside the LM's boundaries, and, canonically - though not always necessarily - oriented towards an open side of the LM and 'ready' to enter.

- Final position or stage:

At the final position, the TR (canonically, the whole of it) is located within the LM's boundaries. A path links TR and LM, the end of which is the LM itself (the series of positions of the TR along the path would topologically mark the 'middle' position).

\section{DYNAMICS}

Force and motion are key features in the basic semantic configuration of into given that the relationship between TR and LM in this utterly basic sense is dynamic in nature: the TR is conceived of as an actively moving entity entering the boundaries of the LM. In other words, the basic dynamic relationship expressed by into is motion of the TR through a force axis or path ending inside the boundaries of the LM.

This axis can take any curve, tilt, direction and angle. Canonically, the force axis is horizontal or vertical (TR upper initial position), as derived from human experience with physical containers - for example, our bodily experience of moving in and out of enclosures on a horizontal axis in the first case or our experience of handling things into or out of containers and preventing them from falling due to gravity forces in the second. However, less prototypically, it may also be conceived of as a vertical axis where the TR is not in an upper, but in a lower position with respect to the LM. This includes cases where the LM is open on its base, for example, injecting hot air into a balloon or screwing a light-bulb into its socket.

- Middle stage:

As for motion features within this force axis, two variations in terms of agentivity are possible: (A) the TR may exert the movement force itself (examples 1,2) or else (B) be externally compelled or attracted towards the LM (3). ${ }^{17}$

- Final stage:

Once inside the LM, the motion of the TR towards the exterior is not completely barred in most cases, given that the LM is open in one of its sides. Nevertheless, the TR's motion within and towards the exterior of the LM may be limited to different extents by the size of the LM itself, of its opening, and the open side (upper, lateral, bottom), which - as the rest of the features described above - has functional side-effects.

\section{FUNCTION}

Both topological and dynamic aspects have a bearing on functional ones. The basic relationship between TR and LM is redescribed functionally in terms of influence and control patterns and affected participants. These tend to be understood, more concretely, in terms of notions like intentions or purposes (canonically, of an animate 
TR with respect to the LM) and effects like protection, reclusion or concealment (usually associated with these intentions).

- Middle stage

Functionally, different degrees of self-control of the TR over the force axis or motion path can be perceived. This control also depends on animacy conditions and can in many cases (when animate participants take a part) incorporate the added value of intention or purpose. Agentivity patterns are tightly related to dynamic aspects but have obvious functional consequences; the two variations described in the middle stage in dynamic terms may be perceived functionally as:

(A) 'Entrance': Self-control of the TR over the motion path. In this case the TR is canonically an animate entity capable of self-motion and/or self-determination e.g. a human being with some intention as in $(1,2) .{ }^{18}$ This relationship is expressed grammatically in intransitive constructions.

(B) 'Insertion': This case denotes a TR with the least possible degree of self-control over the motion path. The TR in this case is fully compelled or attracted by an external force (which may be physical as well as abstract, for example, an inescapable obligation) towards the interior of the LM, as in the case of (3). This relationship is usually expressed grammatically in (simple and complex) transitive constructions (e.g. human beings handling objects).

- Final stage:

The function attributed to the LM in this final stage has a bearing on its effects on the TR. Once the TR is inside the LM's boundaries, some kind of partial control of the LM over the TR may be perceived, the degree of control exerted being strongly dependent upon the limitations (overture size, container size, open side) or restrictions brought in by the LM.

The restriction of TR's movements (e.g. due to the force of gravity, in a container open on its upper side, the TR might find more impediments to exit the LM's boundaries) may be processed functionally as a restriction of its potential action within and with respect to the LM (and any other element within), hence the LM's control. This kind of derived consequence or effect may be labelled reclusion.

Other functional effects usually expected in the relationship are for example protection (the LM may impede the access of other entities) or concealment of the TR or any other element within the LM.

\subsubsection{Contextual information parameters}

Contextual parameters help to establish a more elaborate profile of the kind of TR-LM relationship expressed by ENTRY. Feist (2000) demonstrates the importance of figureground animacy conditions in our conceptualisation of spatial relationships. The role of animacy conditions, especially when dealing with animate TRs, proved a key parameter in identifying usage patterns within the different senses of into. In the case of ENTRY, 
animacy conditions proved crucial in the determination of functional aspects (e.g. control and intention effects described above). Thus, TRs are canonically human beings or objects moved by human beings (e.g. physical hand-size objects, tools, vehicles, etc.). Most LMs, however, are identified as non-animate objects; these may be "real" containers (both fixed and mobile: e.g. house, office, kitchen, room, pool, truck, car) or else conceptualised as such, for example three-dimensional areas perceived as enclosed, but with no boundaries (e.g. woods, trees, cities).

The examples of typical TRs and LMs are related to the nature of the conceptual domains involved in the uses matching each sense of a given preposition. While a particular cluster of domains can be easily identified in secondary senses agglutinating few corpus examples, the vast amount of examples classified into ENTRY yielded a number of general sets, none of which could be considered as particularly representative among the rest. In general terms, physical domains were observed to relate mainly to spaces where people move (enter or leave), for example, buildings and parts of buildings or geographical areas (natural landscapes, cities, counties, etc.). A narrow range of abstract domains was also identified along the examples analysed; these reflect recurrent topics of adult speech especially in areas like police inquiry, education or literature (stories as told in novels and films).

This variety was also found to hold for representative sentential context elements; a general tendency was found, nonetheless, for the combination of 'animate TRs' plus 'non-animate container-like LMs' to collocate with verbs describing the physical motion of the animate agent (intransitive motion/action verbs like go, walk, come, run, wander, etc. or transitive ones like put, place, drive, take, lead, push, throw). These include as well different kinds of verbs with the prefix 'in' (e.g. insert, introduce, investigate). The range of verbs was obviously found to change in the extended uses where metaphoric projections are involved, as the case of the 'shape' metaphor ('shape TR into LM') or the translation metaphor ${ }^{19}$ ('translate TR into LM'): make, shape, convert, turn, transform, change, translate, code, etc.

\subsection{Secondary senses}

The description of the secondary senses provided in this section will only highlight the main modifications, with respect to the primary sense, ${ }^{20}$ that motivate their presence in the network.

Each sense will be introduced by way of the representation of the basic TR-LM configuration plus a series of examples, followed by an explanation of the criteria met plus the description of the main transformations in terms of each construal aspect.

COLLISION and ENCLOSURE are introduced first, as only two of the three criteria are met. TR BECOMES A GROUP and LM CONSTRAINS TR'S CONTROL, where the three criteria are met and the role of metaphor becomes gradually more important, are described next. 


\subsubsection{COLLISION}

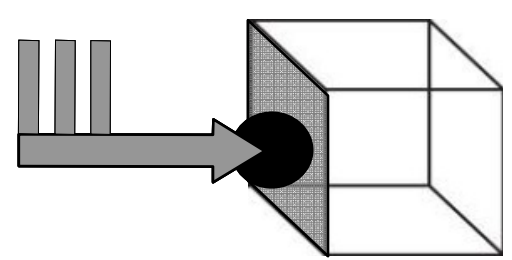

Figure 4. TR-LM configuration of COLLISION

(4) K1B 2906 Fiat's driver was forced to swerve to avoid an on-coming car. As he did so he lost control of the vehicle which rolled over smashed into a lamp-post.

(5) A12 218 Other easily recognised Balanchine signatures can be seen in passages marked Stretto where his dancers stab their toes into the floor as they travel across the stage in posés attitudes devant or à la seconde .

(6) B0B 247 ... they didn't see two boys suddenly come racing round the bend just by Miss Miggs's cottage. The boys, who were bigger than the Brownies, ran full-tilt into them.

(7) JY6 3416 She stabbed an angry finger into his chest.

Into has been suggested as a prototypical case of boundary-crossing preposition (Cf. Tutton, 2009 for a short discussion). This feature, however, is not found in the COLLISION sense and is, in fact, one of the main divergences with regard to the rest of senses. Basically, COLLISION describes a strong movement of the TR towards the LM but, the interior of the LM is never reached by the TR. The particular variations that COLLISION shows with respect to the primary sense can be systematised in terms of criteria $\mathrm{A}$ and $\mathrm{B}$.

Criterion A: The schematic structure of TR-LM configuration of ENTRY has varied in terms of (i) a stronger force-motion component, (ii) a change in the nature of the LM (one of its surfaces receives primary focus, whereas the rest of its structure becomes downgraded) and (iii) the resulting position/state of the TR with respect to the LM. That is, the stage where the TR is expected to be located within the LM in ENTRY does not actually happen in COLLISION; there is therefore a focus on the middle stage of the original TR-LM relationship, so that the last position of the TR with respect to the LM in COLLISION coincides with the end of the middle stage in ENTRY.

Criterion B: The original construal aspect configuration has also undergone several modifications so that, while topological aspects are obviously inherent to the relationship, both dynamic and functional ones become especially salient in this sense. These differences can be redescribed within each multidimensional construal aspect as follows:

\section{TOPOLOGY}

Relevant topological features are concerned with the final position of the TR-LM relationship (interior not reached) and the prominence of the LM's contact point (the grey side in Figure 4) over the rest of its structure (Cf. Criterion A above). 


\section{DYNAMICS}

The dynamic component profiles an extremely strong movement of the TR towards the LM (represented in Figure 4 with a strong-force vector marking the path to be followed) that abruptly stops when the TR collides with the LM.

\section{FUNCTION}

The use of into in this sense is motivated by the force of the movement itself and the impact of the TR on the surface of the LM, which has obvious functional consequences for the whole structure that composes the LM. These consequences, real or perceived by the human being describing the scene, can be measured in terms of affected participants given that, functionally, both TR and (especially) LM become affected.

In terms of topological or dynamic aspects, the situation could be portrayed as a typical case of motion ending in contact. The use of into here is hence motivated by the salient functional effects of the force and motion involved. This use can be considered as hyperbolic inasmuch as there is actual contact between TR and LM, which is strong enough to be perceived as almost trespassing one of the LM's sides, with derived consequences and effects.

This can easily be seen in examples like (4) where two non-animate entities are involved, and where the moving entity does not have control over its motion, or else as well in examples like (5), where the TR is an animate entity controlling motion. Controlled or uncontrolled motion or the shape and structure of the LM are less important here than the effects of motion on both TR and (especially) LM. In both examples the use of into is licensed by functional projections from our human experience of collisions onto the series of real and obvious effects (as in the case of (4), where physical damage consequences for both TR and LM are obvious) or hyperbolic or perceptually relevant effects resulting from strong contact (in (5), in contrast, stabbing a part of the TR into the LM highlights a series of functional consequences for the TR in terms of potential damage of the dancers' toes, but also in terms of the sound derived from this contact with the LM)

Other series of consequences are more easily perceived when animate LMs are involved, as in (6) and (7), where the use of into clearly denotes the consequences (physical in (6) and both physical and psychological, among others, in (7)) derived from the action of the TR with respect to the LM.

\subsubsection{ENCLOSURE}

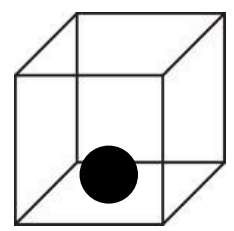

Figure 5. TR-LM configuration of ENCLOSURE 
(8) C85 1479 People were squeezing towards the raised pit to get a better look at the fighting cocks. Two were already straining from the hands of their masters, [...]. Others were in lidded baskets until it was their turn; more tied in sacks; $\underline{\text { a few }}$ buttoned into deep pockets.

(9) K32 2741 Suddenly he was into a totally new environment.

(10) H8J 198 'You are so closed into your fairy-tale world of happy ever after that you'll never bring yourself to form a relationship with a flesh and blood man.

The ENCLOSURE sense portrays the TR enclosed, in a resting position, within the LM. This sense roughly coincides with the primary sense of in described in Navarro (1998: 227-230), where topological, dynamic (force component) and functional aspects yield a balanced construal aspect configuration - hence its location at the centre of the network (Cf. Figure 2).

Functional effects remain basically the same for both prepositional choices; that is to say, both in and into portray, in the ENCLOSURE sense, a closed container that may conceal, confine or protect the TR in different ways. The choice of into, as opposed to in, seems in this case to be inherent to the degree of elaboration of the construed relationship in terms of dynamic and topological aspects. In other words, by using into, the speaker metonymically profiles (and thus emphasises) the endpoint of a previous process of motion, including a path that continues within the LM. Likewise, into further elaborates the TR's final position within the LM, especially at its bottom, as opposed to a less elaborated relationship profiled by in, which basically construes the TR within the LM's boundaries (Navarro, ibid.).

Both criteria A and $\mathbf{B}$ allow the presence of ENCLOSURE in the network. On the one hand, a significant modification upon the original schematic configuration of ENTRY results as the product of a metonymic focus on ENTRY's last stage, which entails as well a selection of the closed-container feature. ${ }^{21}$ On the other hand, as suggested above, a different construal aspect configuration is involved:

\section{TOPOLOGY}

The TR is pictured as already located inside the LM, particularly at the bottom of it and in a resting position. The LM is canonically a closed container.

\section{DYNAMICS}

Previous motion from the outside is implied (although this belongs to a previous stage in the TR-LM relationship not profiled in this sense; in fact, most verbs accompanying this sense profile a static relationship), including a path that continues well into the LM. In the resting position, the forces involved have reached a balance; if the TR is animate, however, there may be potential attempts to break it by reaching the exterior or another location of the LM. There is usually limited motion of the TR within the LM. 


\section{FUNCTION}

Functional consequences meet those found in the final stage described in the primary sense, with effects of concealment, reclusion or protection. These may also be perceived as control restriction effects of the LM over the TR if the latter is animate.

The three examples proposed for this sense range from the more concrete to the more abstract, as far as LMs are concerned. While (8) instantiates the typical TR-LM relationship in physical terms, examples like (9) and (10), where the LM progressively becomes more abstract, are also central. In fact, a series of metaphorical extensions associated with contextual uses within ENCLOSURE underlie a significant part of the examples classified under this sense. ${ }^{22}$

\subsubsection{TR BECOMES A GROUP}

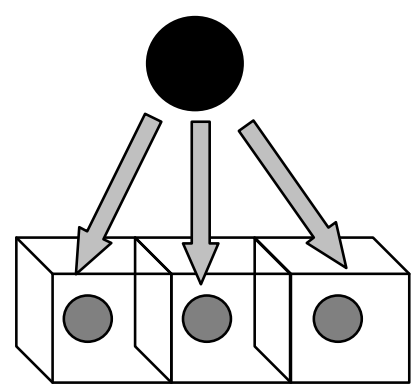

Figure 6. TR-LM configuration of TR BECOMES A GROUP: CLASSIFICATION

(11) EX1 19 Manufacturers were producing bigger and more powerful cars, and the rules, although crude, divided the cars into two categories - heavyweight and lightweight

(12) FAJ 1099 The young men split the cattle into two groups and head off to find pasture.

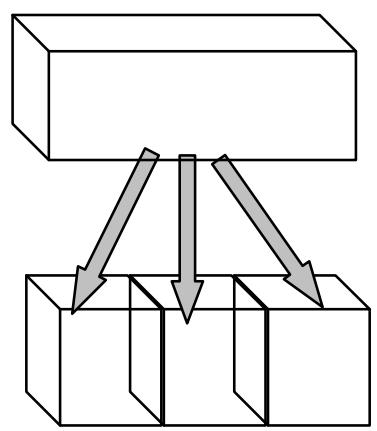

Figure 7. TR-LM configuration of TR BECOMES A GROUP: DIVISION

(13) J13 2320 You take your gram and divide it into twenty-five standard hits.

(14) EAA 585 Conversely, lower achievers who do not segment time into past, present and future (time is now) will write down only one or two goals. 
Criteria A, B and, as a novelty, $\mathbf{C}$ apply in this sense. As for criterion $\mathbf{A}$, a series of modifications (Cf. Figures 6 and 7) upon the basic TR-LM configuration of ENTRY become apparent in terms of number of motion paths and TR-LM transformations. Criterion $\mathbf{B}$ also applies given that there is a special focus on topological and dynamic construal aspects (as opposed to the exclusive emphasis on the dynamic component of ENTRY). Finally, given that a metaphor licenses the existence of this sense (both variations), criterion $\mathbf{C}$ is also met.

Two variations of the TR-LM configuration are possible in this sense: CLASSIFICATION - Figure 6, examples $(11,12)$ - and DIVISION - Figure 7 , examples $(13,14)$. Both of them depend on the nature of the TR and on the particular application of a basic event-structure metaphor.

Concerning the nature of the TR, in the first case the TR is perceived as an undefined (unclassified) collection of entities to be classified into different groups or categories. In DIVISION, however, the TR is a single entity or a mass that is to be divided into different parts that form a whole.

Both variations draw on the changes are movements into bounded regions metaphor, each with a particular set of correspondences. The common ground suggests that any kind of change in the nature of TR is understood as motion of the TR towards the LM. In Figures 6 and 7, therefore, each of the multiple motion paths indicates a metaphorical process of change.

These differences are further developed under each of the multidimensional construal aspects below:

\section{TOPOLOGY}

- Middle stage

Multiple paths provide the blueprint for metaphorical change into the LM. Each of the multiple positions of the TR along each path is redescribed metaphorically as a part of the process of change in which the TR is split (classified or divided) into different parts that, together, compose a whole.

- Final stage

The TR has become a plurality of entities framed within the LM. In the case of CLASSIFICATION, the TR is perceived as arranged within the LM (e.g. cars in categories (11) or cattle in groups (12)). In the case of DIVISION the TR is identified with the LM; that is to say, TR and LM are the same entity in two different stages of change (e.g. unsegmented vs. segmented time in (14)). More specifically, the underlying metaphor allows a conceptualisation of the same entity whose nature has changed from one stage to the other. In it, the TR is identified with the 'before' stage any changes taking place being understood in terms of its motion through each path - , whereas the LM is conceived as the outcome of the process of change, that is, the same entity in the 'after' stage.

It is important to note that the LM is perceived as a group of entities conceived as parts of a whole. More specifically, the LM may be envisaged as a single entity 
composed of different parts - this is typically construed in the case of DIVISION, owing to the identification of the TR with the LM - or else as a collection of individual LMs that, together, are parts of a whole.

\section{DYNAMICS}

CHANGES ARE MOVEMENTS INTO BOUNDED REGIONS finds its application especially in the dynamic component emphasised by this use of into; the description of dynamic features therefore strongly draws on the conceptualisation of change in terms of motion, which profiles the middle stage of the TR-LM relationship:

In basic dynamic terms, in both variations, the movement of the TR - usually caused by an external force or agent - results in a plurality of entities within a group (LM). Thus, the TR's motion 'branches out' into different paths (metaphorically, the TR becomes split into different parts) yielding to the LM (a segmented entity or a group of LMs) in the case of CLASSIFICATION, or yielding (actively creating) different parts of the LM in the case of DIVISION.

\section{FUNCTION}

Although functional aspects remain at the backstage in this sense, the functional rule is that the TR is the controlled (and affected) entity in the relationship expressed by into. One of the dynamic features of this sense is compelled motion, whose metaphorical counterpart is some kind of change imposed by an external agent. In functional terms, the changes in the TR-LM relationship are the product of the intention of the external agent, as in (12), for example, where 'the young men' divide the cattle into two groups in order to have more chances of finding pasture.

The lack of control of the TR over the process of motion/change is reflected grammatically in terms of syntactic and semantic roles associated with transitive patterns. That is to say, the TR is usually found as the patient in passive voice elaborations or as the DO in complex transitive constructions. As a consequence, it is commonly identified as one of the affected entities in the relationship; in the case of DIVISION, where TR and LM are identified as the same entity that becomes divided as a result of the process of change, the LM might also be considered semantically as an effected entity given that a series of new 'items' - 'hits' in (13) or 'past, present and future' in (14) - are created as a result.

\subsubsection{THE LM CONSTRAINS TR'S CONTROL OVER ITSELF}

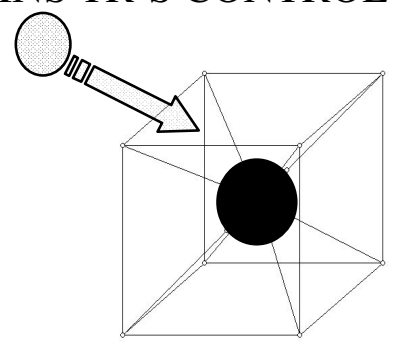

Figure 8. TR-LM configuration of LM CONSTRAINS TR'S CONTROL OVER ITSELF 
(15) K2R 153 It was the perfect example of 'the more you do, the more trouble you get into'.

(16) KS9 217 Some reasons for non-production in mares: 1 Mare not covered — did not come into season; 2 ...

(17) FS0 1140 He was a gentle man by nature, but he would suddenly fall into $\underline{\text { a }}$ depression and lose all confidence in himself.

(18) JYE 1317 The little girl dissolved into giggles, ...

The three selection criteria are also met in this sense:

Criterion A. Variations from the original (ENTRY) configuration can be perceived (Cf. Figure 8) in terms of a special focus on the middle and final stages (similar to Langacker's end-path focus summary scan, only that the middle of the path is also focused upon here) of the TR-LM relationship. This focalisation is actually due to the derived functional implications for the TR once it trespasses the LM's boundaries. ${ }^{23}$

Criterion B. In terms of the configuration of construal aspects, there is in this sense a variation from the original (ENTRY) with a focalisation as well on functional aspects in terms of change of control patterns (the LM gradually gains control over the TR as it gets into its boundaries).

Criterion $\mathbf{C}$. Two combined metaphors in the shape of a metaphoric amalgam (Cf. Ruiz de Mendoza and Galera-Masegosa, 2011) motivate the existence of this sense, and warrant the application of criteria A and B.

The change of control patterns mentioned above is allowed by the presence of a combination of two instantiations of the EVENT-STRUCTURE metaphor: STATES ARE LOCATIONS (bounded regions in space) and CHANGES ARE MOVEMENTS (into bounded regions). This amalgam allows this use of into to construe particular states as containerlike LMs in such a way that any change concerning a new state, condition or situation of the TR (as in (15-18): trouble, season, depression, laughter) becomes the product of the underlying metaphorical interpretation of its motion towards the interior of the LM.

The particular configuration of construal aspects in this sense, being derived from the factors described above, can be schematised as follows:

\section{TOPOLOGY}

The final stage, where the TR is already inside the LM is emphasised.

\section{DYNAMICS}

In terms of source-target domain correspondences, any kind of motion of the TR with respect to the LM in the source domain is conceptualised metaphorically as a change in the target domain.

- Middle stage:

The TR moves along a path towards the LM (metaphorically, the process of change starts). 
- Final stage:

TR enters the boundaries of the LM (metaphorically, a change is taking place). Once inside the LM, there is a heavy restriction ${ }^{24}$ of the TR's movements, both inside the LM and also towards its exterior

\section{FUNCTION}

The functional feature emphasised in this sense is a change of control patterns, from the initial to the final stage describing the relationship between TR and LM. This is the functional consequence/implication of the change described under the Dynamic construal above.

- Initial stage:

The TR shows relative self-control over itself or may have some intentionality towards the change itself.

- Middle stage:

The TR's initial relative self-control or volition diminishes progressively as the middle stage develops (the metaphorical process of change into a new state starts).

- Final stage:

The LM constrains the TR's control over itself (the metaphorical change has taken place); as a consequence, the TR becomes overtly affected by the influence of the LM.

The TR's self-control, intentionality or volition suggested for initial stages can easily be perceived in examples where the TR, being an animate, self-determined being, may have some preconception, intention or volition concerning its entering the LM's boundaries. Although not found in the set of examples randomly extracted from the corpus, it is possible to find examples where the TR, a human being, is willing to enter, say, a positive (or at least, desired) state of body/mind, for example, calmness, concentration, meditation, etc. In these cases, that individual only controls fully the initial stage, an intention and/or an effort (e.g. calming down, focussing on breath) to become focused or, to get into, say, a meditative state. Once that person is in that mental or physical state, leaving it does not fully depend on them, as some physical and mental changes (the LM itself) have taken place as a result of that prior effort.

(15-18) above illustrate cases of a lower degree of TR's self control especially at middle stages. While the degree of self control is nearly nonexistent at this stage in (16) and (17), it is relative in (15) or (18), as the TR might still be able to take some action. For example, the TR in (16) does not have a chance of avoiding 'getting into season' whereas in (15) it might still have a chance of avoiding getting into trouble - by, say, becoming aware that they are still 'in the process of' getting into a situation that might eventually escape their control. In any case the process of change is not as manageable as in the situations described in the previous paragraph, and the resulting state (final stage) remains the same. 
Contextual information parameters are especially important whenever the criterion $\mathrm{C}$ applies because it entails the existence of a metaphor licensing or bringing about a new sense, with a very clear scenario and contextual elements defined. Thus, a note on the role of context is especially relevant here because all the examples found to instantiate uses of this sense (all of them obviously being metaphorical) occur associated with abstract domains (e.g. time, phases/situations in one's life) or else the domain of the human body (in terms of physical or mental states). As it could be expected, TRs are people or animals (although states or feelings themselves can also stand metonymically as TRs) whereas LMs are identified with physical (e.g. 'mare into season') or mental (e.g. depression) states, feelings, habits, or momentary situations (e.g. 'trouble'). Finally, the typical verbal elements found are causative (grammatically speaking, this use of into is a good locus for the caused-motion construction), occurrence or activity verbs (e.g. force, turn, come, sink).

\section{Conclusion}

This paper has illustrated, by way of the analysis of into, the process of the derivation of polysemic units departing from the extraction of corpus examples to their classification and arrangement within a multidimensional semantic network (Navarro, 1998, 2006; Silvestre-López, 2009).

The two methodological aims set for this study have been attained with the help of the methodological procedures proposed. In the construction of the network, the study of contextual parameters has helped to learn behavioural patterns of into across the pieces of discourse analysed. Among them, the analysis of TR-LM animacy conditions of into has proved a key factor in determining the nature of the senses showing highlighted functional aspects. Setting an identifiable series of features and criteria for sense identification, discrimination and classification have, on the one hand, proved an important methodological guide in the overall example analysis and classification process and, on the other hand, helped to keep the number of senses in the network to a minimum, while forming a coherent set.

Consequently, the theoretical and methodological considerations presented here are expected to become a valuable tool in the process of derivation of multidimensional semantic networks for any particle (preposition or adverb) within the model.

The multidimensional polysemy framework is a descriptive model and its results reflect language use tendencies within a linguistic community. On the one hand, this kind of description allows establishing systematic contrasts between particular prepositions in terms of intralinguistic (e.g. into vs. in and to) and crosslinguistic (e.g. English in/on/at vs. Spanish en (Navarro, 2006)) equivalences derived from the processes and ways in which languages encapsulate perceived spatial relationships and their effects (Silvestre-López, 2009). This line of research becomes especially relevant for the case of into, as a compound preposition; further studies must in this regard analyse the semantic contrasts arising from the use of into, in and to in English. On the other hand, these studies become especially useful when applied the field of translation, 
and especially in ESL learning contexts with different kinds of learners, a line of work that is currently being developed by the research group to which the author belongs.

As any linguistic approach on prepositional semantics, the work presented here is not free from limitations. One of them concerns the scope of the analysis of into: while the parameters used to derive the network may be applied in different studies (all of them being framed within the multidimensional polysemy model), the scope of the results concerning into is more restricted. Although the sample of corpus examples analysed seems wide enough to allow for the whole spectrum of senses and to establish generalisations in terms of use, in strict terms, the results of this study are limited to a closed set of randomly extracted examples from a much wider database, the BNC.

An additional limitation is related to the subjective component that is obviously present in this study. Albeit the methodological tools allow for a series of relatively objective steps, the manual analysis of examples and their classification still relies partially on the researchers' interpretation. In order to reduce the effect of subjective interpretations, the series of decisions on the classification of each of the senses that involve an interpretative component have been made in consensus with a group of researchers trained in the model. Nonetheless, the process of classification might benefit from additional insights derived from cross-analyses conducted by a further series of annotators.

Finally, this study only includes a basic description of the essential components of different senses associated with into in discourse. The different kinds of rearrangements derived from the application of the selection criteria and the role of metaphor as a meaning extension device, left unaddressed in this paper for space reasons, will be developed in more detail in other forthcoming works.

\section{Notes}

1. Research sustained by the Fundació Bancaixa Castelló-UJI, grant P1-1A2010-14.

2. Because the three dimensions have a bearing on our active perception and construction of spatial concepts, these three aspects might also be labelled dimensions of perceptual space. Alternatively, given that they also take a part in construal formation, they may also be referred to as aspects or dimensions of construal. In this paper I shall use both terms interchangeably.

3. This label is used by Navarro (2006) instead of force-dynamics.

4. An illustration of the structural layout and construal configuration of ENTRY, the protoconcept of into can be found under sections 3.4.1. (Figure 3) and 3.4.2.

5. The reader might like to access the network proposed for into (Figure 2 under section 3.3) where senses are already distributed.

6. Navarro (2006: 176) makes reference to processes of "profiling of particular elements" or "partial sanction" of the protoconcept. These processes are considered in this study as particular instantiations of metonymies. Metonymy is an extremely comprehensive tool that can not only embrace these two processes (Cf. section 2.2.2.), but may also become a key element in that metonymic projections (e.g. focalisations) based on the original structure of the primary sense help to avoid network overpopulation. 
7. These have implicitly guided sense derivation under this approach, but have not yet been overtly systematised under a coherent set.

8. Owing to the nature and objectives set for this study (especially in terms of methodological repercussion) a series of theoretical considerations are also included under the methodology section.

9. 'Proper sense status' depends on the selection criteria devised to attain AIM 2.

10. The research conducted for this study also yielded results concerning a well-defined set of sense profiles that, when rearranged into proper senses, contributed to an enriched variety of context-specific usage tendencies within each sense. It also allowed tracing the behaviour of metaphor along the network in terms of representative metaphorical extensions of ENTRY (meaning extension through metaphor) and metaphoric projections within secondary senses. A brief description of the sense profiles is provided below but, owing to space restrictions, nor context-specific usage tendencies nor metaphorical extensions are treated in this paper.

11. Examples of proper senses are provided in further sections.

12. The presence of metaphor here does not trigger a change in the original structure or construal aspect configuration. This is therefore a case of metaphoric extension, not licensing (criterion C).

13. These parameters were analysed in all senses of the network, but they will only be accounted for under the description of the primary sense and when necessary in the description of secondary senses so as not to exceed the length of this paper.

14. BNC standard notation: [text code] [line number].

15. The schematic representations of the TR-LM relationships presented in this paper are not intended to resemble other kinds of traditional representations like, for example, Johnson's (1987) image schemas or Talmy's (1988) force-dynamics. Any coincidence has been avoided on purpose because the senses described in this study are more comprehensive, that is to say, image schemas and force-dynamics are actually two of the components underlying the nature of protoconcepts and derived senses

16. The stages that are not relevant in a particular construal aspect are not made explicit.

17. Reference to examples given will be provided from now on in parenthesised numbers.

18. The reader may note that although the speaker in (2) is a person with an explicit intention (i.e. going into hospital for an operation, so that there is an implicit aim, a function of the LM that the speaker seeks to enter), the use of 'I've got to go' implies, in dynamics terms, a subtle external compelling element which, functionally speaking, might be translated into a need to regain good health that triggers the intention itself. The degree of self-control in (2) is, owing to the added compelling force element, lower than that in (1).

19. As suggested earlier, these metaphors are the object of description of a different paper.

20. Although it is common to find different nodes and levels of derivation (Navarro, 1998, Silvestre-López, 2009) the proper secondary senses of into were all eventually found to stem from the central node.

21. The exclusive metonymic focus on ENTRY's last stage renders previous stages as the base against which the ENCLOSURE sense (as the last stage focussed upon) is profiled. Thus, while different stages in the TR-LM relationship can be distinguished in the configuration of the rest of senses, only one stage defines the construal aspect configuration described below.

22. The treatment of metaphorically-extended uses falls beyond the scope of this study and will therefore not be addressed here. Nonetheless a brief example is provided here for illustrative purposes: Particular uses include examples of the kind 'to be into something' showing that a human TR is interested in a particular activity (LM). The use of this expression 
metaphorically construes the TR within the LM (i.e. eagerly involved with(in), as if physically surrounded or cosily enclosed in it and not willing to change its state by leaving its boundaries), as the result of a previous process (motion through previous stages) in which the TR progressively 'became more interested in' such an activity.

23. This added implication is reflected in the diagram in terms of enriched structure focussing on the final stage of the relationship.

24. The notion of heavy restriction is also represented in the TR-LM configuration layout (Figure 8). This exemplifies one of the cases where the original structure of ENTRY may be perceived as being enriched, as new factors that were potentially possible, but not canonically effected, in its original structure come into play in this new sense.

\section{References}

Brugman, Claudia Marlea (1980): The Story of Over. M.A. Thesis. University of California at Berkeley.

Coventry, Kenny and Garrod, Simon (2004): Saying, Seeing, and Acting: The Psychological Semantics of Spatial Prepositions. New York: Psychology Press.

Deane, Paul (2005): "Multimodal spatial representation: On the semantic unity of Over". In B.

Hampe and J.E. Grady, eds., From Perception to Meaning. Image Schemas in Cognitive Linguistics. Berlin: Mouton de Gruyter, 235-282.

Dewell, Robert (2005): "Dynamic patterns of CONTAINMENT'. In B. Hampe and J.E. Grady, eds., From Perception to Meaning. Image Schemas in Cognitive Linguistics. Berlin: Mouton de Gruyter, 369-393.

Evans, Vyvyan (2009): How Words Mean: Lexical Concepts, Cognitive Models, and Meaning Construction. Oxford: OUP.

Feist, Michelle (2000): On In and On: An Investigation into the Linguistic Encoding of Spatial Scenes. Ph.D. Dissertation. Northwestern University, Evanston, IL.

- (2004): "Talking about space: A cross-linguistic perspective". In K.D. Forbus, D. Gentner and T.Reiger, eds., Proceedings of the Twenty-sixth Annual Meeting of the Cognitive Science Society. Malwah, NJ: Lawrence Erlbaum Associates, 375-380.

Geeraerts, Dirk (2007): "Family resemblances, radial networks and multidimensional models of meaning”. In M. Losada, P. Ron, S. Hernández and J. Casanova, eds., Proceedings of the XXX AEDEAN Conference. Huelva: Publicaciones de la Universidad de Huelva.

Geeraerts, Dirk, Grondelaers, Stefan and Bakema, Peter (1994): The Structure of Lexical Variation: Meaning, Naming and Context. Berlin: Mouton de Gruyter.

Grady, Joseph (2007): "Metaphor". In D. Geeraerts and H. Cuyckens, eds., Handbook of Cognitive Linguistics. Oxford: OUP, 188-213.

Johnson, Mark (1987): The Body in the Mind. Chicago: University of Chicago Press.

Lakoff, George (1987): Women, Fire, and Dangerous Things: What Categories Reveal about the Mind. Chicago: University of Chicago Press.

Langacker, Ronald Wayne (1987): Foundations of Cognitive Grammar. Volume I: Theoretical Prerequisites. Stanford: Stanford University Press.

Navarro, Ignasi (1998): A Cognitive Semantics Analysis of the Lexical Units At, On, and In in English. Ph.D. Dissertation. Castelló: Publicaciones de la Universitat Jaume I. 
(2006): "On the meaning of three English prepositions". In I. Navarro and N. Alberola, eds., In-roads of Language, Essays in English Studies. Castelló: Publicaciones de la Universitat Jaume I, 167-179.

Navarro, Ignasi and Gösser, Birgit (2011): "Semantic configuration of the spatial concept Behind in English". Studia Universitatis Petru Maior - Philologia 10: 204-209.

Peña, María Sandra and Ruiz de Mendoza, Francisco José (2009): "The metonymic and metaphoric grounding of two image-schema transformations". In K. Panther, L. Thornburg and A. Barcelona, eds., Metonymy and Metaphor in Grammar. Amsterdam/Philadelphia: John Benjamins, 339-361.

Ruiz de Mendoza, Francisco José and Galera-Masegosa, Alicia (2011): "Going beyond metaphtonymy: metaphoric and metonymic complexes in phrasal verb interpretation". Language Value, 3(1): 1-29.

Silvestre-López, Antonio José (2009): Particle Semantics in English Phrasal and Prepositional Verbs: The Case of In and On. Saarbrücken: VDMVerlag.

Talmy, Leonhard (1988): "Force dynamics in language and cognition". Cognitive science 12: 49-100.

Tutton, Mark (2009): "When In means Into: Towards an understanding of boundary-crossing In". Journal of English Linguistics 37: 5-27.

Tyler, Andrea and Evans, Vyvyan (2003): The Semantics of English Prepositions. Spatial Scenes, Embodied Meaning and Cognition. Cambridge: CUP.

Vandeloise, Claude (1991): Spatial Prepositions: A Case Study from French. Chicago: Chicago University Press.

(2003): "Containment, support, and linguistic relativity". In H. Cuyckens, R. Dirven and J.R. Taylor, eds., Cognitive Approaches to Lexical Semantics. Berlin: Mouton de Gruyter, 393-425. 